\title{
EchoGéo
}

$38 \mid 2016$

Varia

\section{Les concours de l'enseignement : s'y préparer et réfléchir aux passerelles didactiques entre géographie scolaire et géographie scientifique}

\section{Sophie Bresc-Litzler}

\section{(2) OpenEdition}

\section{Journals}

Electronic version

URL: https://journals.openedition.org/echogeo/14814

DOI: $10.4000 /$ echogeo.14814

ISSN: 1963-1197

\section{Publisher}

Pôle de recherche pour l'organisation et la diffusion de l'information géographique (CNRS UMR 8586)

Electronic reference

Sophie Bresc-Litzler, "Les concours de l'enseignement : s'y préparer et réfléchir aux passerelles didactiques entre géographie scolaire et géographie scientifique", EchoGéo [Online], 38 | 2016, Online since 13 December 2016, connection on 01 August 2021. URL: http://journals.openedition.org/ echogeo/14814; DOI: https://doi.org/10.4000/echogeo.14814

This text was automatically generated on 1 August 2021.

EchoGéo est mis à disposition selon les termes de la licence Creative Commons Attribution - Pas d'Utilisation Commerciale - Pas de Modification 4.0 International (CC BY-NC-ND) 


\title{
Les concours de l'enseignement : s'y préparer et réfléchir aux passerelles didactiques entre géographie scolaire et géographie scientifique
}

\author{
Sophie Bresc-Litzler
}

\section{Introduction}

\author{
« Enseigner exige de déterminer l'enjeu \\ méthodologique, civique et didactique d'un \\ savoir en histoire ou en géographie $»^{1}$.
}

Cette phrase extraite du rapport du jury de la session rénovée du concours doit attirer l'attention car elle rappelle les attentes et les méthodes à appliquer pour préparer les nouvelles épreuves des concours de l'enseignement (CAPES externe). L'enjeu méthodologique renvoie ainsi aux méthodes utilisées à l'acquisition d'un savoir. Cette acquisition passe par la lecture approfondie des ouvrages de référence des grands historiens ou géographes des questions qui sont au programme. À propos de ces enjeux méthodologiques, l'étudiant doit se demander quelles sont les finalités de la transmission de ces savoirs aux élèves. Autrement dit, nous pouvons nous demander par exemple pourquoi former aujourd'hui des enseignants à la géographie thématique des Mers et océans. La finalité de cette question est de fournir aux futurs enseignants les clés d'un savoir disciplinaire qui pourrait répondre à la maitrise générale des programmes comme aux futures questions des élèves (par exemple de $5^{\text {ème }}$ et de $2^{\text {nd }}$ ). Les mers et les océans sont au cœur des enjeux du développement durable de la planète et interrogent les futurs citoyens, consommateurs, et acteurs économiques. Nous retrouvons ici la finalité civique de l'enseignement secondaire. C'est aussi ce principe de formation à la citoyenneté qui a conduit à la mise en place en 2016 des E.P.I. (enseignements pratiques interdisciplinaires). 
2 Pour enseigner, il faut aussi se soumettre aux attentes des épreuves des concours de recrutement et avoir des réflexes disciplinaires et intellectuels. Ce sont des épreuves exigeantes qui nécessitent une maîtrise rigoureuse des savoirs disciplinaires et des compétences didactiques (maintenant évaluées aux épreuves écrites et orales du CAPES dit « rénové ») pour être capable de transmettre en classe des savoirs raisonnés et d'en comprendre les finalités civiques. Au-delà de la lettre du rapport du jury qu'il est indispensable de connaitre, cet article a pour objectif de proposer aux candidats des concours du CAPES d'histoire-géographie des réflexions méthodologiques (techniques de rédaction, de schématisation spatiale, constitution de banques de données utiles à la mémorisation) pour aborder de façon constructive et efficace leur préparation aux épreuves. La lecture approfondie des rapports parus soit dans la revue Historiens et géographes soit sur le portail en ligne du ministère de l'Education Nationale Eduscol reste indispensable pour comprendre les attentes du jury.

Ces rapports établis à l'issue des épreuves orales paraissent en général en septembreoctobre de l'année qui précède le concours de l'année suivante (parution en septembre octobre 2016 pour la session 2017). Les membres du jury participent à leur rédaction, le rapport est présenté par le président du jury ( $M$. Vincent Duclert). La composition du jury reflète la structure des institutions universitaires et éducatives (IGEN, IA-IPR, Professeur des Universités, Maître de conférences, PRAG, professeur de classe préparatoires et professeur agrégé et certifié exerçant dans le secondaire). La lecture des rapports du jury sur les dix dernières années montre qu'ils sont de plus en plus détaillés et qu'ils offrent des conseils à la fois précis et pratiques comme par exemple le déroulement concret d'une épreuve orale. Ces rapports sont ainsi des sources d'informations publiques gratuites très appréciables aussi bien pour les candidats que pour les préparateurs à ces mêmes concours.

4 Nous souhaiterions avoir ici une approche complémentaire de celle des manuels ou des ouvrages parus spécifiquement ${ }^{2}$ pour la préparation aux concours en offrant aux candidats des outils et des ressources pour acquérir des connaissances (en lien avec les questions du programme) : il faut comprendre ainsi les techniques de «transposition didactique des savoirs et de notions » attendues par les jurys et correcteurs et qui seront utilisées en pratique face aux élèves après la réussite au concours.

5 Nous nous appuyons sur une expérience professionnelle à la fois en tant qu'enseignante dans le secondaire (depuis 2005) et préparatrice au concours du CAPES (épreuves écrites et orales, de 2014 à 2016) en construisant notre propos à partir d'exemples concrets, des conseils, des méthodes de travail et de rédaction pour être capable de se distinguer à l'écrit. L'application de ces méthodes au travail personnel de l'étudiant durant l'année de préparation sur des exemples judicieux apportent une plus-value à sa copie (exemples de schématisation sur des études de cas, méthodes de mémorisation...) et on ne peut aborder les épreuves écrites sans la technique maîtrisée de la transposition didactique (mobilisation d'éléments d'actualité en lien avec les thèmes et les programmes, connaissance des programmes du secondaire - techniques appliqués ici aux deux questions de géographie présentes pour la session 2017 du CAPES externe : «Mers et océans » et « la France des marges »). 


\section{Les exigences des nouveaux concours du CAPES externe}

\section{La « bivalence histoire-géographie »}

Le CAPES externe est un concours qui a profondément évolué. La première mutation du concours se traduit tout d'abord par la «bivalence histoire-géographie » (Duclert, 2014) Les deux disciplines sont aujourd'hui à égalité et les candidats sont invités à ne pas négliger une discipline par rapport à l'autre. Il ne faut pas aborder le concours avec un tropisme disciplinaire en histoire ou en géographie. Cela oblige donc les candidats à acquérir une bonne connaissance des méthodes et des savoirs aussi bien en histoire qu'en géographie. Cela peut impliquer parfois une remise à niveau sur des notions fondamentales; ainsi en géographie par exemple, il faut être capable de maitriser et définir clairement les termes de mondialisation, d'interface, de centralité, de périphérie, de marge comme en histoire les grands repères historiques et les bases bibliographiques. Les rapports du jury le rappellent suffisamment, avec la nécessité de la «maîtrise des savoirs d'histoire et de géographie » (Duclert, 2014, p. 99).

7 Cette bivalence histoire-géographie se retrouve également dans les conseils indiqués par le Conseil Supérieur des Programmes parus dans le Bulletin Officiel de l'Éducation Nationale (B.O.E.N) : «L'enseignement de l'histoire et de la géographie doit être envisagé dans la complémentarité entre les deux disciplines : les équipes de professeurs mettent en œuvre les questions programmées dans l'une et dans l'autre de manière équilibrée, à parts égales, en n'hésitant pas à souligner les apports de l'histoire à la géographie et réciproquement » (B.O.E.N., 2015).

8 La bonne pratique du métier d'enseignant a besoin d'une maîtrise des deux disciplines, qui est parfois négligée par des enseignants eux-mêmes. Cela explique l'évolution professionnalisante de la bivalence dans les épreuves écrites et orales du CAPES pour répondre aux risques de déséquilibre dans la mise en œuvre des programmes ou la progression des enseignants entre histoire, géographie, éducation morale et civique (EMC). Pour préparer les concours de l'enseignement, il faut donc se construire une solide culture générale en Histoire et en Géographie et des méthodes fondamentales du cycle de formation universitaire (rédaction, dissertation, étude de document, problématique et analyse synthétique). Ainsi le rapport du jury de la session 2015 décrit les techniques universitaires à maîtriser pour le commentaire de document: «La première partie, dite "analyse critique", est un commentaire composé du dossier de documents proposé, selon les règles universitaires qui régissent cet exercice scientifique. Un commentaire composé n'étudie pas les documents les uns après les autres, mais les présente de manière critique puis les rapproche et les confronte afin de faire ressortir leurs informations principales, les représentations de leurs auteurs ou institutions d'origine, leurs fonctions et leur autorité, leurs éventuelles postérités et usages historiographiques, etc. C'est agir ici en historien ou en géographe " (Duclert, 2015, p. 5). Les candidats doivent donc être aussi compétents et bien formés en histoire qu'en géographie. 


\section{Construire une culture générale reliée aux enjeux de la transposition didactique}

Les connaissances fondamentales doivent composer une solide culture générale, mobilisable et applicable rapidement. Ce point n'est pas nouveau et a souvent été exigé des candidats bien avant la session rénovée du CAPES. Dans la version ancienne du concours, les connaissances de base et d'actualité étaient demandées notamment à l'oral de l'ancienne épreuve intitulée ESD (épreuve sur dossier) ${ }^{3}$. Le candidat doit être capable de se constituer, à l'aide d'une banque de données facilement réalisable, une culture générale qui lui permette de comprendre pourquoi tel élément d'actualité est et peut devenir un objet d'enseignement. Les questions des programmes en lien avec des thématiques actuelles appellent clairement les candidats à réfléchir à la finalité de ces savoirs (notamment civiques) et les conduisent à se demander avec quelle démarche, par quelle mise en pratique et sur quels supports ils peuvent travailler pour transmettre un tel savoir. Auparavant, le candidat se construisait une culture générale personnelle souvent d'abord par goût et ensuite par la nécessité d'être au courant de l'actualité et d'être un citoyen éclairé et averti face à des élèves. Avec le CAPES rénové, cette exigence tient toujours mais se systématise. Ce qui est fondamentalement innovant et différent ${ }^{4}$, c'est de se demander (par exemple en lisant la presse ou en regardant les journaux télévisés et les documentaires) quelles sont les sources exploitables ou quels sont les documents utiles à connaître pour répondre aux exigences de la transposition didactique et finalement aux objectifs de l'orientation professionnelle qu'ont pris les concours de l'enseignement ${ }^{5}$. Concrètement, une lecture régulière de la presse permettra au candidat de se construire une culture générale tout en s'interrogeant sur leur exploitation didactique, en en tirant des exemples précis et mobilisables afin de se constituer une banque de données personnelles et actives, utile à la fois pour préparer les épreuves du concours et par la suite mobilisable dans leur carrière professionnelle. Se constituer une telle banque de données exige de la rigueur et de la méthode, mais aussi une forme de modestie universitaire préalable, qui permet un travail continu et éclairé; ces techniques sont fondées sur l'ambition d'une formation professionnelle à la transmission autant qu'à la connaissances disciplinaire ; nous pouvons retrouver quelques unes de ces techniques dans la deuxième partie de notre présentation.

10 La géographie est une science de plus en plus mobilisée par les journalistes et les médias. Cette discipline rencontre aujourd'hui un plus large public. Des émissions comme le Dessous des cartes contribuent à diffuser des connaissances scientifiques géographiques. Les émissions radiodiffusées comme CulturesMondes ou Planète Terre diffusent largement les actualités et les avancées récentes de la recherche en géographie et en géopolitique. Le succès relatif des atlas en librairie offre des synthèses cartographiques sérieuses et souvent produites et commentées par des spécialistes ${ }^{6}$. L'audience de ces émissions et la multiplication des "livres-atlas" sont la matérialisation des passages et des transferts à l'œuvre entre une géographie de scientifiques et des lecteurs-auditeurs de plus en plus intéressés par les apports d'une réflexion fondée sur l'étude de l'espace et de ses représentations ${ }^{7}$. La géographie est populaire dans la culture française et dans la presse dont la tradition qui consiste à aborder dans un premier temps les thèmes géopolitiques perdure tant dans la presse écrite que dans l'audiovisuel. Les productions cartographiques présentées dans ces 
médias parfois animées et diffusées sous forme de vidéos (par exemple les cartes animées sur le site du Monde) sont construites en respectant les conventions de la sémiologie graphique. Ainsi aujourd'hui de nombreuses connaissances sont acquises à l'aide de ces nouveaux médias (site de journaux, documentaires ou les émissions de radio). On y retrouve parfois les spécialistes des thèmes aux concours. Cela aide à la connaissance bibliographique. Aussi est-il profitable de suivre des émissions scientifiques ou de vulgarisation en lien avec les questions traitées comme celles de France Culture (La Fabrique de l'Histoire, Concordance des Temps ou Planète Terre et son blog...). Les émissions de radio ont le grand avantage d'être podcastables avec une recherche thématique facile. Ce type de média favorise clairement les transferts de connaissances scientifiques entre les spécialistes et les étudiants préparant les concours.

De même concernant la question Mers et océans, nous suggérons les nombreux documentaires passés sur la chaîne ARTE depuis trois ans comme l'excellent Qui contrôle la mer? (ARTE, 2015), ou Les Enjeux de la sécurité maritime (ARTE, 2015), Adieu thon bar saumon (soirée théma ARTE, 2014), Surpêche la fin du poisson à foison (ARTE, 2014), Quand les océans deviennent acides (ARTE, 2014), Océans en Danger (ARTE, 2014), Alerte rouge sur le thon (ARTE, 2014), Armes chimiques sous la mer (ARTE, 2014), End of the line l'océan en voie d'épuisement (2012)... On trouve aussi d'autres très bons documentaires sur d'autres chaînes publiques comme Le Port du Havre Un monde de démesure (France 5, 2014), Cargo La face cachée du fret (France 5, 2016)... Nous invitons par exemple à réfléchir sur l'utilisation pédagogique de ces sources numériques; le site d'ARTE Future (http:// future.arte.tv/fr/categories/environnement), propose un étonnant exemple d'enquête interactive: Le Monopoly des océans, produit par ARTE et Mobydok: http:// oceans.arte.tv/monopoly/\#/chapter/1. Le suivi de l'actualité est aujourd'hui plus facile grâce aux entrées thématiques ou par les recherches sur les sites de presse comme FranceTVinfo (http://www.francetvinfo.fr/images/videos/) pour le traitement de l'actualité de France 2 et de France 3, Le Monde, Le Courrier international. Enfin les synthèses excellentes de l'émission Le dessous des cartes (par exemple La Pêche la fin de l'abondance, 2011; La pollutions des mers, 2012...), l'accès gratuit aux synthèses en vidéographies de l'AFP accessibles sur la plateforme YouTube: https:// www.youtube.com/playlist?list=PLAo5j5U8Vm-t4hk38FQ-mjn0lpP6EG4Ex. Nous ne fournissons évidemment pas de liste exhaustive pour l'utilisation des médias dans la formation. Tel n'est pas ici notre propos. Il faut aborder toutefois le concours avec une connaissance de ces nouveaux médias, de leur possible utilisation en cours, voire d'utilisation interactive (webdocu...), intégrée raisonnablement dans une progression ou en EPI, et qui est un support de mise en activité ou d'étude de cas ou un complément de réflexion (sans bien sûr se substituer au cours). De plus il faut garder un œil critique et une expertise méthodologique sur ces médias que permet la formation en histoire et en géographie (par exemple en montrant aux élèves comment croiser l'information entre les différents types de médias en complétant les données ou les chiffres avec d'autres sources et noter comment une information est souvent réutilisée par les médias eux-mêmes avec une certaine « circularité » de l'information).

\section{Saisir les programmes pour en comprendre les enjeux didactiques}

12 Parmi les nouvelles exigences du concours figure explicitement la bonne connaissance des programmes et des manuels du secondaire ${ }^{8}$. Les candidats exploreront les manuels 
du secondaire comme des outils leur permettant de comprendre les enjeux et les finalités de la transposition didactique de savoirs. Avant la session du CAPES rénové, les jurys pouvaient attendre des candidats qu'ils connaissent les grands axes et thématiques du programme en leur posant notamment des questions à la suite des épreuves orales. Aujourd'hui le candidat ne peut se contenter d'une simple connaissance des grandes lignes du programme ; il lui faut se plonger dans les manuels, connaître les chapitres de l'enseignement du secondaire en lien avec les questions au programme, voire anticiper les passerelles entre les questions aux concours et le contenu des programme du secondaire pour préparer aussi bien les écrits que les oraux. Les deux épreuves orales du CAPES mettent en effet l'accent sur la connaissance des programmes du secondaire et plus particulièrement l'épreuve orale 2 intitulée "Analyse de situation professionnelle " (ASP). Cette épreuve contient deux ensembles de document: "Le premier ensemble documentaire contient des documents directement liés à la séquence d'enseignement proposée : un extrait des programmes, la fiche EDUSCOL, un exemple de traitement prélevé dans un manuel (incluant cours et étude de cas) [...] Le deuxième ensemble contient des documents offrant au candidat de nouvelles ressources pour cette séquence d'enseignement. Les documents à l'appui de cette seconde partie d'exposé sont au nombre de deux, un écrit issu de la recherche ou de la vulgarisation savante, et un texte de réflexion civique et morale ${ }^{9}$. Cette épreuve orale confirme l'orientation "professionnalisante " des savoirs et des connaissances évalués à l'oral. D'ailleurs le dossier de documents de cette épreuve ASP « est introduit par un titre qui se présente de la manière suivante : "Enseigner........ en classe de ......" ${ }^{10}$. Il est ici intéressant de constater une certaine évolution des épreuves orales du CAPES. Dans les années 2000, le jury évaluait par exemple les connaissances épistémologiques des candidats avec l'ancienne épreuve nommée ESD (épreuve sur dossier). Dans un rapport datant d'octobre 2000 le jury mentionnait pour cette épreuve cinq champs de sujets : «l'épistémologie, l'historiographie et l'histoire de la pensée géographique, les finalités et les objectifs de l'enseignement de l'histoire, de la géographie et de l'éducation civique, l'organisation des programmes des collèges et des lycées et l'usage des documents dans les disciplines et leur enseignement ${ }^{11}$. Avec les nouvelles épreuves orales du concours, nous pouvons ainsi observer un glissement de l'évaluation des compétences qui maintient les connaissances de nature épistémologiques mais qui signale l'importance de la réflexion sur la pédagogie et la didactique se rapprochant bien d'une "professionnalisation» des concours de l'enseignement ${ }^{12}$.

Une bonne connaissance des programmes du secondaire ne saurait être acquise entre les épreuves écrites et orales. Les programmes du secondaire sont en effet denses, variés et riches. Les entrées en géographie fonctionnent par grands thèmes (La France des villes) et aussi par enjeux (nourrir les hommes, l'eau une ressource essentielle). Il existe des formes de répétitions entre les cycles d'apprentissages et les différents niveaux du collège et du lycée. Il est ainsi nécessaire d'avoir du temps pour comprendre les articulations entre les niveaux et les connaissances propres à chaque thème pour chaque classe. C'est un travail qui s'effectue tout au long de l'année par touches successives en se familiarisant progressivement avec les manuels, en les utilisant et en les exploitant. Une bonne méthode pour maîtriser les programmes serait de se "promener » dès le début de l'année et régulièrement dans les manuels en identifiant les passerelles entre les questions aux concours et les programmes du secondaire. 
Qu'entendons-nous par ce terme de "passerelle»? Les programmes actuels de la géographie scolaire abordent à l'aide de nombreuses entrées des thématiques explorées par la géographie universitaire. Nous y retrouvons en effet les notions de mondialisation, d'environnement avec le développement durable et la gestion des ressources, de géopolitique notamment dans les programmes de terminales mais aussi des thématiques qui relèvent de l'aménagement avec les territoires de proximité (programmes de $1^{\text {ère}) . ~ C e s ~ t h e ̀ m e s ~ a p p a r t i e n n e n t ~ a u s s i ~ a u x ~ g r a n d s ~}$ mouvements de la géographie universitaire (géopolitique d'Y. Lacoste, développement durable d'Y. Veyret par exemple) ${ }^{13}$. Il est donc intéressant pour un futur enseignant d'identifier dans sa préparation au concours, les "passerelles» entre la géographie scolaire et universitaire. Les concours et les programmes tendent toujours à établir ce lien épistémologique entre secondaire et université. Si la réactualisation des programmes du secondaire paraît fréquente aux enseignants et oblige à faire des efforts de mise en place de nouveaux programmes, cela se comprend en lien avec ces nouveaux concours : fluidité des études de cas avec multi-support, attention aux études transversales et interdisciplinaires. Les concours accompagnent cette modification de l'enseignement de la géographie: on demande des compétences pour analyser des études de cas innovantes, tout autant que la maîtrise des concepts. On demande de maitriser des mises en pratique d'étude de cas dont les contours disciplinaires moins rigides permettent de les mettre en relation avec d'autres disciplines ou permettent une mise en pratique par groupes d'élèves. Dans sa pratique professionnelle, l'enseignant est en réalité un passeur de savoir scientifique ; il explore les notions, il maîtrise les grands concepts d'une discipline et il en connaît son histoire, son évolution et ses avancées. Il est en définitive celui qui favorise la transmission d'un savoir géographique scientifique à des jeunes publics scolaires et assure à son échelle la continuité de la discipline géographique. Nous proposons ainsi dans les deux tableaux ci-dessous des exemples qui permettraient au candidat d'identifier ces passerelles entre ces deux géographies; l'une scientifique qui s'exprime par la voix des universitaires et des chercheurs dont les travaux de recherches portent sur les thèmes de programmes au concours et l'autre scolaire illustrée par les programmes officiels repris par les manuels du secondaire. Ces deux tableaux sont appliqués aux questions de géographie suivantes : "Mers et océans » et " la France des marges». À l'aide de ces deux tableaux, il s'agit de montrer au candidat comment réaliser le travail de repérage dans les programmes du secondaire. Une bonne connaissance des programmes n'implique pas uniquement un apprentissage linéaire de leur contenu. Il s'agit d'avoir déjà face à ces programmes et aux fiches Eduscol une approche problématisée et de se demander quels sont les savoirs disciplinaires à maîtriser et quelles sont les techniques de la transposition didactiques mises en évidence dans ces programmes et dans les manuels (identification des questions liées à des études de cas par exemple et questionnement sur le choix des documents qui illustrent les études de cas). Les tableaux ci-dessous mettent en évidence les grandes thématiques à connaitre pour les questions au programme. Ces thématiques problématisées ont été identifiées à partir des bibliographies indiquées sur le site de l'ENS de Lyon Géoconfluences et à partir de la présentation des questions par le jury ${ }^{14}$. À partir de ces thématiques, nous avons indiqué les thèmes et les chapitres qui s'y rapportent dans les programmes $d u$ secondaire. Les notions essentielles pour la préparation du concours et pour la transposition didactique de l'épreuve 2 sont également référencées. En recensant les passerelles entre concours et programmes du secondaire, nous comprenons aussi que la 
maîtrise des savoirs disciplinaires et les enjeux de la transposition didactique ne sont pas les mêmes entre le collège et le lycée. La mondialisation n'est ainsi évidemment pas abordée de manière aussi approfondie entre les programmes de Quatrième et de Terminale : les notions ne sont pas définies de manière aussi détaillées et les documents choisis n'ont pas le même niveau de complexité (un choix d'images comme des photographies ou images publicitaires pour les quatrièmes, des textes longs pour les terminales). En parcourant les programmes et les manuels, le candidat peut ainsi plus facilement saisir les différences entre les niveaux. Le premier tableau est construit à partir des anciens programmes pour les classes de collèges. En italique nous avons ajouté les nouvelles entrées liées à la réforme des programmes du collège qui est rentrée en vigueur à la rentrée 2016. Nous nous sommes appuyés sur le BOEN spécial $n^{\circ} 11^{15}$. Le candidat aura donc une vision des transitions qui seront à l'œuvre pour l'année prochaine sur l'ancienne question «Mers et océans» (illustration 1). En revanche pour le deuxième tableau sur la "France des marges", nous présentons uniquement les nouvelles entrées et nouveaux chapitres indiqués par le Conseil Supérieur des Programmes dans le BOEN (illustration 2). Ce tableau est donné à titre indicatif pour aider les candidats à préparer la nouvelle question de géographie. Les programmes de lycée n'ont pas changé; ils ne sont pas encore concernés par la réforme.

Illustration 1 - Thèmes passerelles : des programmes aux notions fondamentales

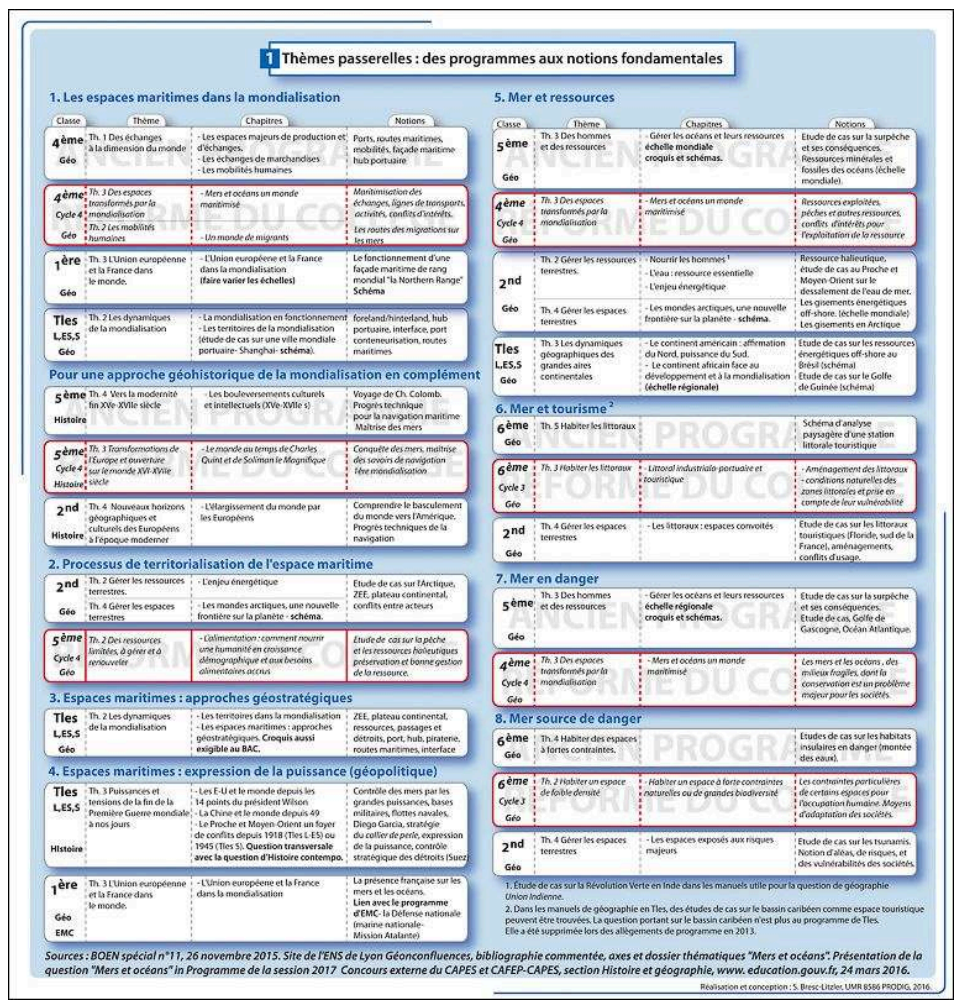


Illustration 2 - Proposition de thèmes passerelles : question la France des marges.

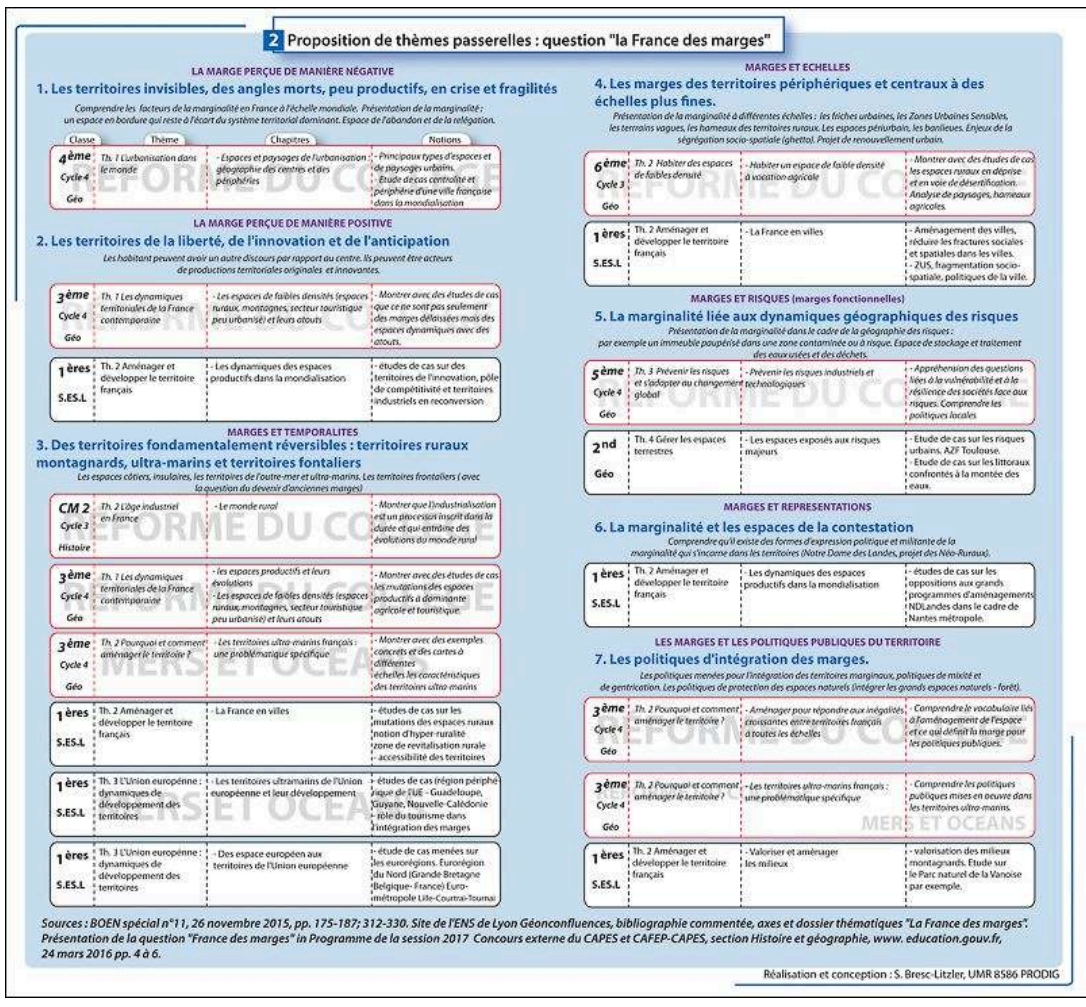

15 Ce travail dans l'apprentissage et la bonne connaissance des programmes du secondaire est utile à plusieurs titres. Il permet au candidat de revoir les notions fondamentales des savoirs disciplinaires en parcourant des définitions synthétiques, en identifiant des schémas d'organisation spatiale et en s'appuyant sur les organigrammes offerts par les manuels pour mémoriser ces notions et comprendre efficacement leurs relations de causes à effets.

Ces deux tableaux permettent aux candidats d'avoir une approche pragmatique dans l'utilisation des manuels scolaires afin de répondre au mieux aux exigences de l'analyse critique et de la transposition didactique. Il existe une sorte de cheminement méthodologique identifiable et reproductible pour d'autres thématiques de la question. Prenons l'exemple facilement réalisable et compréhensible des routes maritimes dans le fonctionnement de la mondialisation. L'illustration 3 propose une méthode à suivre pour identifier les notions, savoir les définir, en comprendre les enjeux et intégrer ces éléments de connaissance de manière problématisée dans une réponse pour l'analyse critique ou la transposition didactique. Cette figure est en réalité une méthode de travail préparatoire aux écrits et aux oraux du concours. L'identification des notions pourrait être celles qui seraient mobilisées pour la partie 2 de l'épreuve 2 écrite. Connaître les programmes du secondaire et le lien entre le sous-thème (ici route maritime) et la fiche Eduscol correspondante (ici soit le programme de 4 ème, soit le programme de Terminale) offre la possibilité d'anticiper le niveau qui sera mentionné dans la transposition didactique de l'épreuve 2. De la même manière, le volet «représentation » a une double finalité : faciliter la mémorisation de données avec l'appui d'images en les reliant à des notions (ici hinterland et maritimisation des échanges) et se confronter à des documents que l'on pourrait éventuellement retrouver dans l'analyse critique de l'épreuve 2 écrite. Nous avons aussi voulu montrer dans cette 
infographie que le candidat pouvait développer des compétences propres et valorisables dans le développement de sa réponse dans l'analyse critique. Nous avons volontairement choisi un document issu du sujet de l'épreuve 2 de la session $2015^{16}$. Il s'agit de la photographie de Yann Artus Bertrand du port de Yangshan. Nous montrons ainsi comment exploiter pédagogiquement une telle image en faisant un schéma d'analyse paysagère comme le mentionne la correction détaillée offerte dans le rapport du jury session $2015^{17}$. Nous pouvons ici rappeler que l'analyse paysagère est une approche scientifique qui reste centrale en géographie car elle permet de montrer comment les hommes façonnent l'espace et les milieux pour en faire justement des territoires c'est-à-dire des espaces appropriés par les sociétés humaines. Nous retrouvons aussi dans l'approche paysagère les travaux de Georges Bertrand et notamment le concept de "géosystème " dans lequel le paysage est une interface démontrant les interactions entre les sociétés humaines et les milieux (Ciattoni et al., 2005). L'étude des paysages est fréquemment pratiquée dans la géographie scolaire car elle offre le moyen de faire comprendre aux élèves que l'espace en géographie est de moins en moins naturel et de plus en plus anthropisé (les études montrent souvent des zones littorales fortement aménagées). Le paysage est aussi une image (photographie ou dessin) qui facilite la compréhension et la perception de phénomènes parfois complexes pour des jeunes publics. Le schéma d'analyse paysagère est une technique didactique promue par la géographie universitaire classique parfaitement adaptée à la géographie scolaire.

Cette iconographie présente enfin des outils de mémorisation (données sous forme d'infographies extraites d'un article du Monde, références bibliographiques et auteurs à connaître ${ }^{18}$ ) pour mobiliser rapidement ses connaissances qui viendront illustrer efficacement des idées à développer soit pour une dissertation (épreuve 1 écrite) soit pour étudier des documents (analyse critique épreuve 2 écrite). 


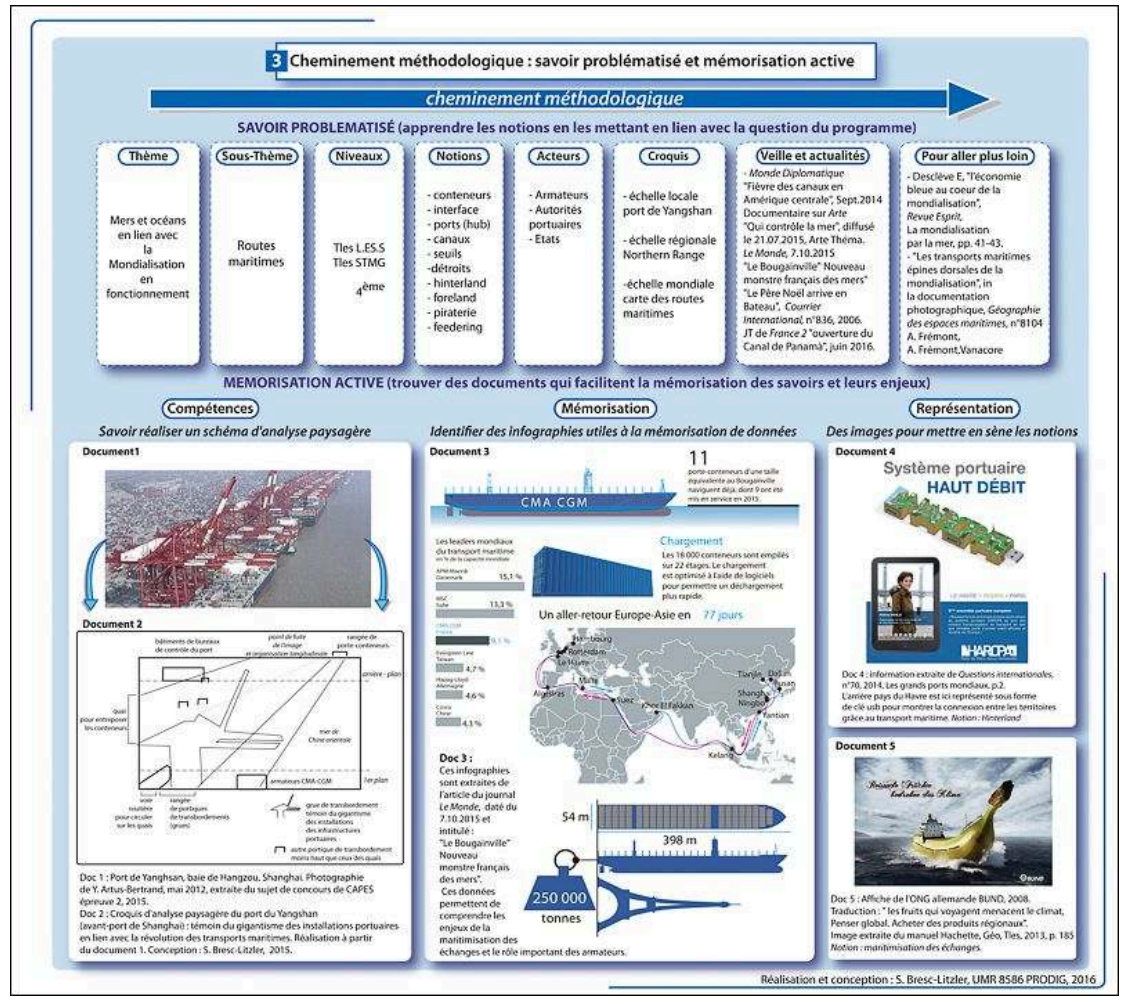

Ce travail approfondi peut être prolongé par un travail actif de recherche et de mémorisation de données scientifiques à partir des programmes du secondaire en approfondissant notamment les études de cas. L'étude de cas est une innovation didactique introduite dans les années 2000 en classe de Seconde puis généralisée dans les programmes actuels du collège et du lycée depuis 2009. Cette technique de l'étude de cas s'appuie sur une démarche inductive qui vise à construire une réflexion pédagogique en partant d'un « cas » particulier pour arriver à une compréhension plus générale d'un phénomène. Selon Gérard Granier : « l'étude de cas n'est pas un simple exemple illustratif puisque c'est par son intermédiaire que le professeur doit faire comprendre à ses élèves les notions ou les concepts géographiques qui sont au programme » (Granier, p. 11-12). L'étude de cas est aujourd'hui fréquemment pratiquée en classe et des didacticiens se sont intéressés à l'élaboration d'outils efficaces permettant une bonne mise en œuvre pédagogique de cette technique inductive. Ainsi dans leur ouvrage, Yannick Mével et Nicole Tutiaux-Guillon(2013) expliquent clairement comment conduire une étude de cas en classe (notamment à l'aide d'un tableau construit par rubriques hiérarchisées). Selon eux : « l'étude de cas correspond à un cas réel, complexe, qui permet de travailler raisonnements et concepts ; (...) Une étude de cas nécessite du temps, temps de compréhension et de mise en œuvre des concepts, temps nécessaire pour conduire des raisonnements, temps d'argumentation et de discussion, temps de synthèse final " (Mével et Tutiaux-Guillon, 2013). Ainsi les études de cas développées dans les manuels permettent de comprendre les exigences de la transposition didactique (notions à connaître, exemples à intégrer éventuellement dans une narration, choix des documents par les auteurs du manuel pour répondre à une problématique, lecture des questionnements proposés par les auteurs à partir des documents) et de compléter sa culture générale en comblant parfois des lacunes personnelles pour répondre à l'exigence de la «bivalence histoire- 
géographie ». L'illustration 4 ci-dessous est un exemple d'un travail plus précis sur une étude de cas. Nous avons identifié un sous-thème de la question "Mers et océans » : les ressources halieutiques. En explorant les manuels, nous avons pu trouver dans le programme de Seconde une étude de cas intéressante portant sur une activité peu connue pratiquée sur le littoral chinois : l'algoculture ou la culture des algues. Cette étude de cas développée dans le chapitre Les littoraux des espaces convoités permet de comprendre la notion de conflits d'usage qui peuvent nuire au développement d'un système de culture marine. L'étude entre dans la question du concours car elle montre les menaces environnementales et économiques qui pèsent sur la gestion et la préservation d'une ressource halieutique massivement cultivée et consommée dans l'aire régionale asiatique (la Chine est le premier producteur mondial d'algues avec près de 10 millions de tonnes en 2010). Les documents de la page du manuel ont été réalisés à partir d'un article scientifique (Litzler, 2012). L'intérêt de notre infographie est de montrer le passage de l'article scientifique (géographie universitaire) à l'étude de cas du manuel en montrant notamment la simplification de la carte qui est plus accessible pour un public scolaire (géographie scolaire). Cette étude de cas issue d'un manuel (Janin, 2014) synthétise convenablement l'article scientifique (extrait de l'article et un extrait du Courrier International) et offre un accès rapide à des données facilement mémorisables pour le candidat au concours avec un exemple spatialisé. Les correcteurs apprécient grandement les synthèses simples et proprement illustrées. Nous pouvons voir aussi la transmission efficace de données scientifiques pour des élèves de classe de Seconde et comprendre à l'aide d'un exemple les problématiques du chapitre (aménagement du littoral, préservation des activités traditionnelles de la mer, littoraux convoités et conflits d'usages), illustrant les passerelles entre problématiques issues de la géographie scientifique et contraintes de la géographie scolaire. 
Illustration 4 - De l'article scientifique à l'étude de cas dans le manuel : l'exemple de l'algoculture dans le golfe de Bohai

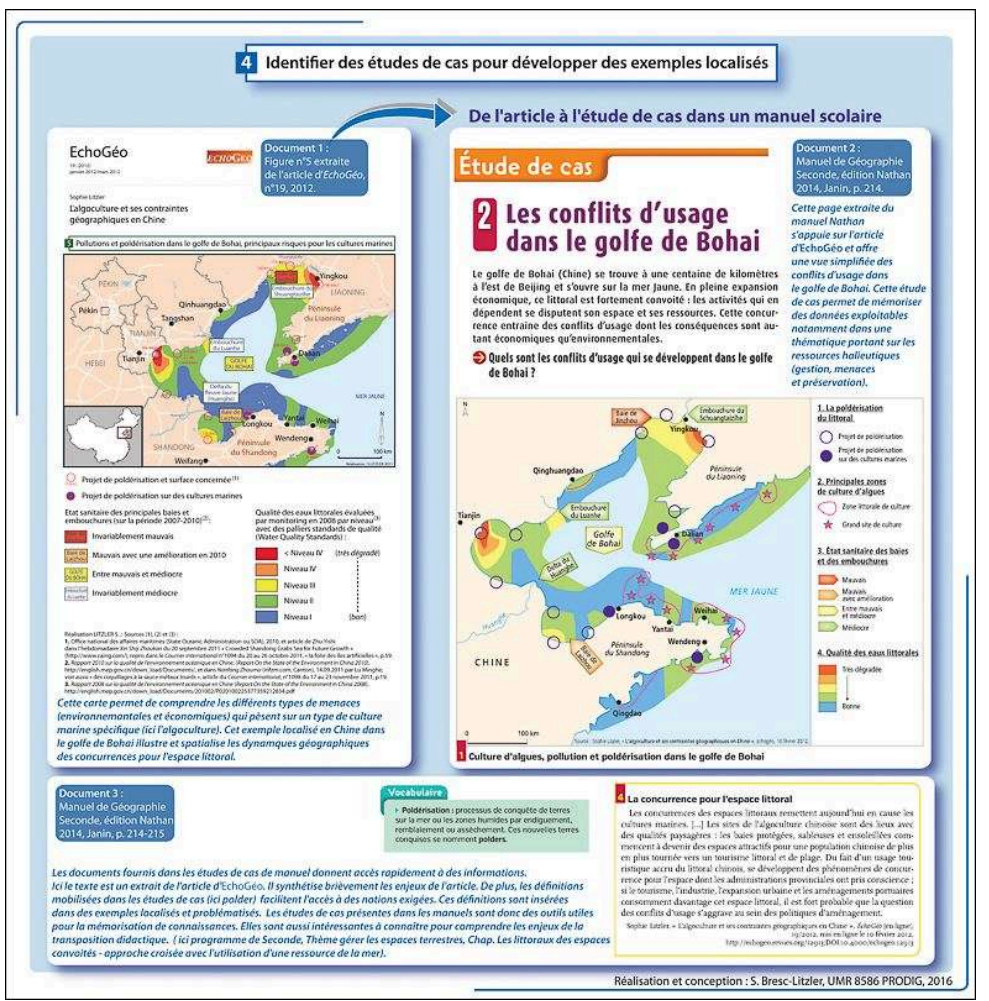

19 La fréquentation studieuse, la compréhension et la maîtrise des programmes du secondaire permet aussi de répondre aux exigences des épreuves en travaillant tout au long de la préparation et en ne distinguant pas la préparation écrite de celle de l'oral ${ }^{19}$. Le travail d'analyse des pages de manuel permet aussi de se projeter dans une situation professionnelle en se demandant par exemple : comment les rédacteurs des manuels ont-ils interprété les programmes officiels ? Pourquoi avoir choisi telle problématique? Pourquoi avoir trouvé ces documents? Permettent-ils de répondre aux enjeux de la problématique choisie ? Les questions qui accompagnent l'étude de cas sont-elles enfin pertinentes $?^{20}$ La connaissance fine des programmes et le travail problématisé autour des notions présentes dans les programmes et en lien avec les thématiques des questions au concours constituent une excellente technique de préparation et sont des compétences à développer pour préparer le CAPES rénové. Un travail personnel qui parcourt le contenu des manuels et identifie des études de cas intéressantes procure ainsi au candidat une bonne connaissance pratique des contenus scientifiques (notions, connaissances) et pédagogiques (croquis, schéma) enseignés dans les différents niveaux du secondaire. Cette méthode peut aider à préparer le volet 2 de l'épreuve écrite 2 à savoir « Exploitation à un niveau donné ", en complément d'un travail de lecture des fiches Eduscol et d'une réflexion sur les modalités du passage d'un contenu scientifique à un contenu pédagogique ${ }^{21}$.

Ces méthodes de travail synthétisées dans ces quatre figures permettent ainsi de «combiner la maîtrise scientifique et l'intelligence pédagogique ${ }^{22}$ et montrent en images et en texte commenté comment l'histoire et la géographie sont enseignées en pratique. 


\section{Des méthodes pour maîtriser les savoirs scientifiques et les transmettre pédagogiquement}

21 Nous souhaiterions dans cette deuxième partie présenter les techniques et les méthodes servant à se créer une banque de données personnelles pour gagner en efficacité dans la mémorisation des savoirs fondamentaux. Puis nous verrons comment répondre aux exigences de la schématisation en présentant les techniques de rédaction pour intégrer ces plus-values dans les copies aux concours.

\section{Constituer une banque de données personnelles pour mémoriser les savoirs.}

Les éléments d'actualité sont indispensables à la compréhension des épreuves de géographie contemporaine qu'il faut savoir synthétiser et aller chercher dans des supports en ligne, dans les revues scientifiques, sur internet (accès gratuit comme dans Domino, portail de la documentation électronique du service commun de la documentation de l'université...), dans les documentaires présents sur les sites vidéo et dans la veille des documentaires thématiques (source d'information et support de mémorisation). La géographie scolaire explore en effet de nombreuses thématiques en lien avec l'actualité contemporaine (géopolitique des grandes puissances, conflits en mer et espaces maritimes) ce qui en fait une discipline pédagogiquement attractive pour l'enseignant et les élèves tout en contribuant à l'objectif de formation de futurs citoyens.

Nous proposons ici aux candidats, une méthode de travail fondée sur l'utilisation de ces nouvelles ressources qui pourront être utiles par la suite en classe devant les élèves. Les questions de géographie sont fondamentalement ancrées dans les thématiques et les problématiques des sociétés actuelles. Les spécialistes des questions venant de la recherche et de l'enseignement supérieur sont présents dans ces médias, dans les documentaires, les émissions, les débats ou les interviews. Les médias et internet sont aujourd'hui des sources d'informations utiles que le candidat peut utiliser pour venir compléter des lectures, pour approfondir des thématiques vues en cours ou parcourues dans les manuels. Cette sensibilisation à l'offre d'information provenant des nouveaux médias et des contenus disponibles en ligne est utile d'une part parce qu'elle permet de mémoriser des données à l'aide de supports différents et d'autre part parce que l'enseignement d'aujourd'hui dans les collèges et les lycées fait appel à l'utilisation de vidéos ou de sites internet. Les équipes d'histoire-géographie des lycées et des collèges s'organisent pour acquérir des ressources réactualisées. Ce travail s'effectue avec l'aide des documentalistes du C.D.I en s'abonnant à des revues (par exemples la Doc photo, Carto, l'Histoire), en achetant des documentaires en DVD et en offrant la possibilité aux élèves de parcourir la presse avec les abonnements à Europress par exemple. La géographie est une discipline qui a une relative diffusion médiatique ; elle évolue ellemême rapidement en lien avec cette ouverture ; l'offre de documentation des manuels peut s'avérer parfois vite dépassée et dépend du renouvellement opéré dans les établissements scolaires. Elle nécessite alors le recours à des données réactualisées très souvent disponibles dans les médias et sur internet. Il faut garder une méthodologie scientifique indispensable au choix de ces documents, à la critique de la mise en forme des données et à la vérification de ce qui est avancé. La figure ci-dessous (illustration 5) 
présente ainsi sous forme d'infographie synthétique une typologie des ressources disponibles et leur mode d'utilisation grâce à trois entrées : méthodes, pratiques et exemples. Nous avons donné une liste indicative d'exemples qui peut être complétée. Nous souhaiterions montrer au candidat que ce travail de recherche dans des nouvelles ressources doit rester rigoureux et méthodique et permet de se tenir au courant de l'actualité : il soutient ainsi une culture générale personnelle à partir de ces données et de ces références scientifiques et institutionnelles.

Illustration 5 - Techniques et méthodes pour utiliser de nouvelles ressources

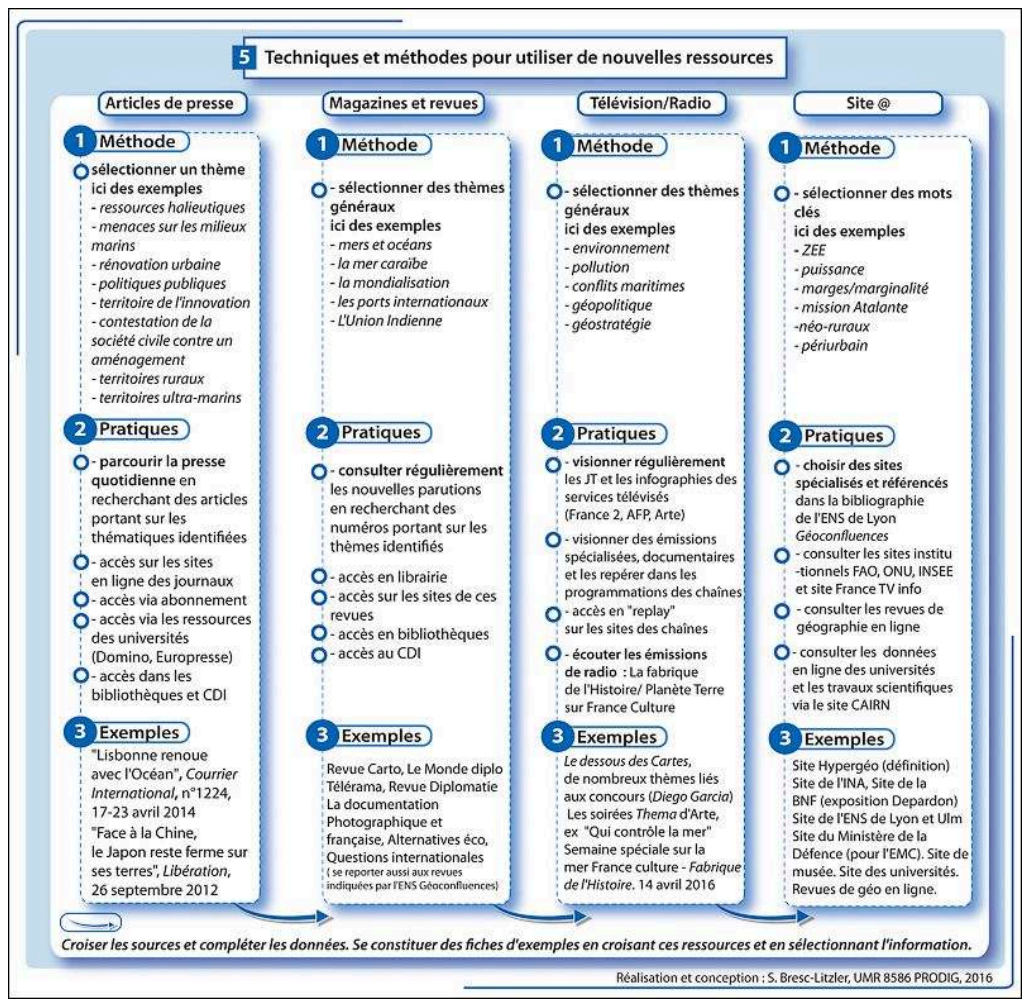

24 Nous aimerions enfin montrer que l'abord de ces données (par la sélection de sites officiels, par la recherche à l'aide de mots-clés précis, par la connaissance rigoureuse et scientifique des notions, par sa distance aussi critique vis-à-vis des outils numériques) donne au candidat la possibilité d'être souvent autonome dans son apprentissage des connaissances. Il s'agit en effet d'être capable aussi de trouver les sources primaires d'informations et de compléter activement les lectures des manuels au concours. Il existe des sites d'institutions officielles qui délivrent des informations gratuites et faciles d'accès : site de l'ONU, de la FAO, de l'INSEE, de l'ONPES... Beaucoup de ces sites institutionnels sont d'ailleurs référencés dans les bibliographies des concours (site de l'ENS de Lyon, Géoconfluences) ${ }^{23}$. Ces sites institutionnels publient des rapports réactualisés et téléchargeables gratuitement utiles à condition d'en dégager les grandes lignes avec l'étude rapide du sommaire, le relevé d'exemples et de chiffres pour développer un exemple ou pour illustrer une idée extraite d'un article scientifique, l'identification de cartes originales qui peuvent être réappropriées en schéma. L'illustration 6 montre par exemple à partir d'un rapport officiel de l'ONU ${ }^{24}$ comment un candidat peut produire une carte efficace pour montrer les évolutions de la piraterie aux larges des côtes africaines. Ce travail peut se faire aussi en groupe de travail, partageable en ligne sur les réseaux sociaux. 
Illustration 6 - Mobiliser des connaissances dans des sources primaires d'informations : l'exemple de la piraterie

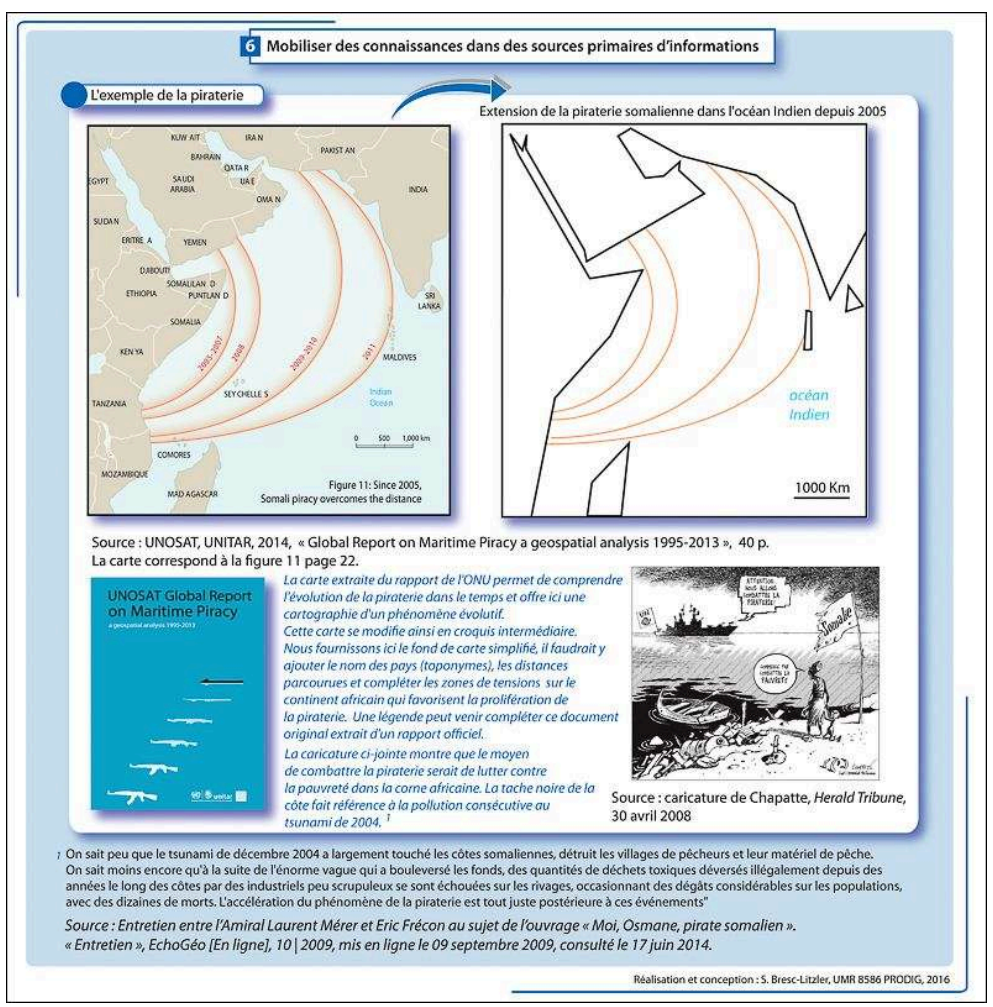

Cette infographie à laquelle nous avons ajouté une caricature en lien avec le sujet (piraterie et pauvreté) montre que le candidat peut explorer les rapports officiels des grands sites institutionnels pour avoir accès à des connaissances. La démarche est active et de ce fait facilite aussi le travail de mémorisation en allant rechercher des informations, en les croisant avec d'autres sources (ici l'article référencé d'EchoGée ${ }^{25}$ ) et en fournissant des efforts pour les schématiser et les mettre en lien avec des problématiques de la question (ici piraterie, pauvreté, diffusion d'acte illégal en mer, routes maritimes de la mondialisation victimes de la piraterie et moyens de lutter efficacement contre).

La schématisation est une technique utile à la mémorisation des connaissances et permet aussi de répondre aux exigences des épreuves écrites du concours en spatialisant notamment la réflexion.

\section{Comment répondre aux exigences disciplinaires de la schématisation à des fins pédagogiques?}

Le rapport du jury de la session 2014 offre au candidat un corrigé de l'épreuve 1 "composition" en géographie. Le sujet s'intitulait "Minorités et recompositions territoriales en Amérique du Nord (Canada, États-Unis, Mexique) ${ }^{26}$. La lecture de ce corrigé est éclairante car le jury présente ici des consignes claires pour répondre aux exigences de la schématisation (croquis intercalaires et croquis de synthèse) pour les épreuves d'admissibilité écrites (illustration 7). 
Illustration 7- Critères pour répondre aux exigences de la schématisation

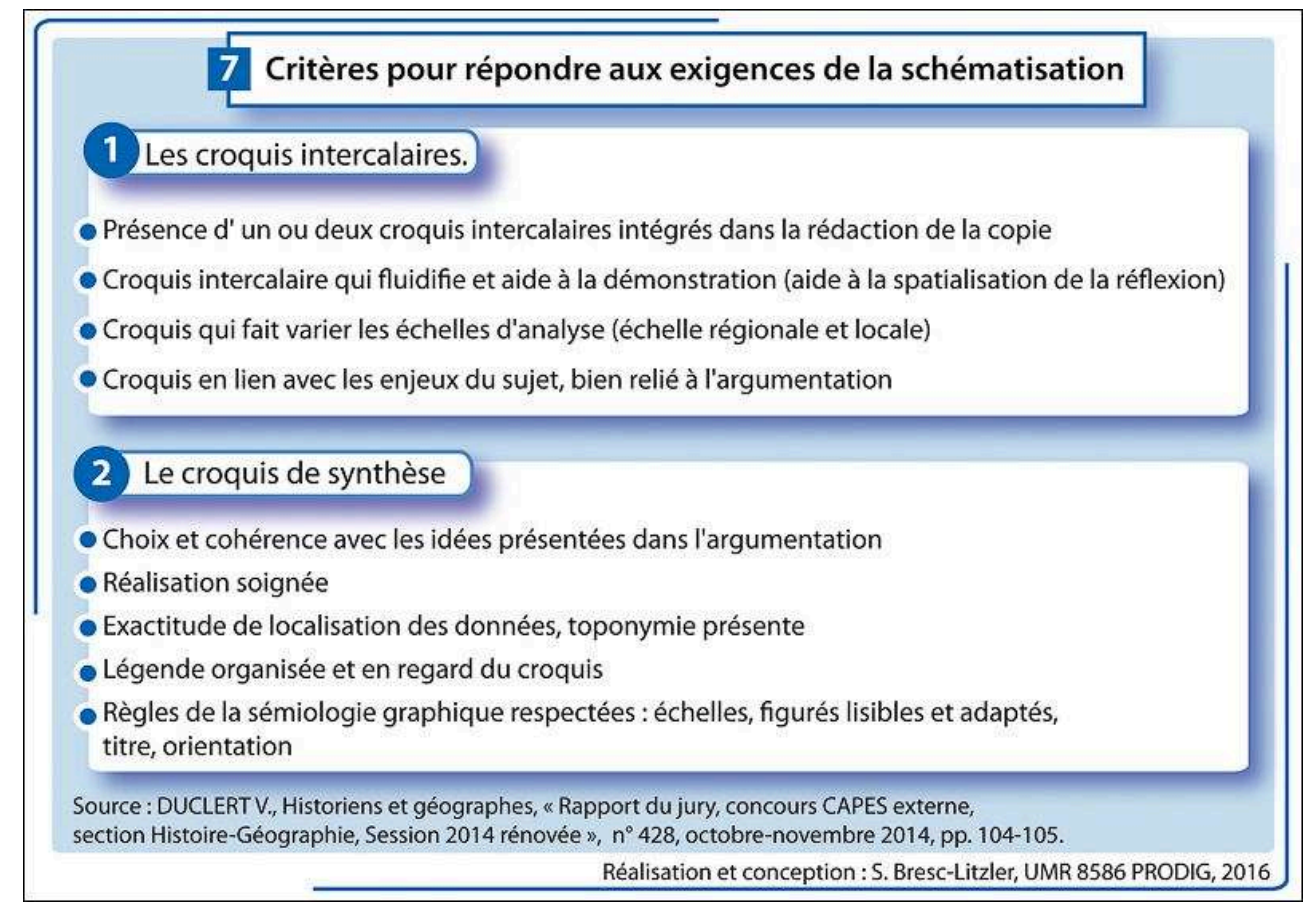

À partir de ces critères, nous proposons aux candidats deux exemples de schématisation spatiale pour réaliser des croquis ou schémas intermédiaires à deux échelles régionales différentes (1. Asie de l'Est, 2. Moyen-Orient; illustration 8) ${ }^{27}$. Il s'agit ici de raisonner en géographe et de proposer une autre représentation des territoires étudiés. À l'aide d'échelles d'analyses différentes, l'objectif didactique est de proposer un autre découpage de la carte pour en comprendre l'organisation et ses dynamiques. Roger Brunet dans un article de la fin des années 1990 interrogeait justement la finalité de la géographie en interrogeant «l'art de la découpe » en géographie (Brunet, 1997; Sierra et Bonnet-Pineau, 2016). Cette schématisation intercalaire est bien présente pour illustrer l'organisation de l'espace, pour montrer les systèmes spatiaux et leurs articulations. Cette exigence pédagogique de la schématisation spatiale et $\mathrm{du}$ découpage à l'échelle régionale sont des savoirs disciplinaires incontournables et trouvent ici leur transposition didactique dans la géographie scolaire. 
Illustration 8 - Répondre aux exigences de la schématisation

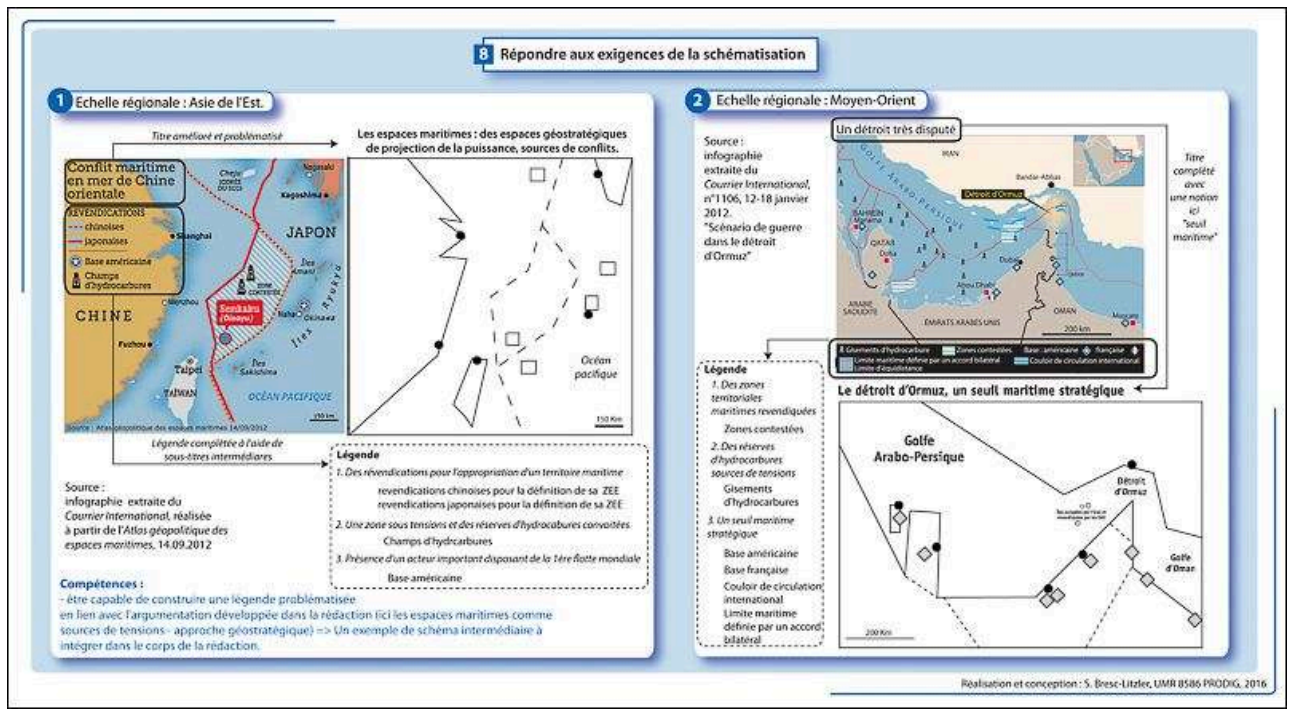

Les croquis intercalaires privilégient davantage les échelles régionales ou locales. Nous avons ainsi identifié dans la presse des cartes utilisables à des échelles intermédiaires en lien avec les questions du programme des concours (ici mers et océans). Les médias fournissent un nombre important d'infographies sur des thématiques du concours (espaces maritimes protégés, espaces maritimes sources de tensions, détroit convoité mais aussi étude sur les dynamiques périurbaines des villes, rénovation des centres villes urbains). Les avantages de ces cartes grands publics sont nombreux : elles sont problématisées en lien avec une actualité récente; elles sont souvent facilement reproductibles sous la forme de schéma géométriques comme nous l'avons fait dans l'illustration 8 ; les légendes de ces cartes sont brèves et mémorisables; le candidat peut facilement se les réapproprier en complétant et en structurant la légende (hiérarchisation des informations avec des sous-thèmes conformément aux critères d'évaluation du rapport du jury) ; les figurés sont simples et suffisamment explicites pour être repris. Le candidat vérifie alors le respect des règles de la sémiologie graphique (figuré de surface, ponctuel et linéaire, bonne superposition des informations avec par exemple les hachures verticales ou horizontales tracées à la règle et respectant les écarts entre les traits, effort de localisation et toponymie). Il doit également indiquer sur son schéma le titre, l'échelle, l'orientation et de les numéroter (s'il y en a plusieurs) afin d'y faire référence dans le cours de son développement (les légendes de ces croquis intermédiaires peuvent être utilisées dans différentes parties du développement).

30 Aux schémas construits à partir des données issues de la presse peuvent s'ajouter les schémas d'organisation spatiale proposés dans les manuels du secondaire. Le candidat pourra ainsi se réapproprier des schémas (avec la méthode décrite ci-dessus) portant par exemple sur les enjeux des ressources halieutiques dans le bassin d'Arcachon, sur les conflits d'usages au sein de la réserve naturelle marine de Cerbère-Banyuls ou encore sur les nouvelles dynamiques de protection des espaces marins comme le Parc national des Calanques de Marseille (entre valorisation et gestion des milieux dans le premier parc périurbain de France) (Husken et Bervas, 2011, p. 101, 109, 111). Les revues de cartographie et les manuels de préparation aux concours sont aussi des sources riches et utiles pour se « fabriquer » ses schémas personnels. 
Nous souhaiterions ici rappeler aux candidats deux conseils utiles donnés dans les rapports du jury. Les croquis intercalaires insérés dans la rédaction d'une composition et au fil de la réflexion montrent des capacités de synthèse valorisées. Ils ne doivent toutefois pas être récités et insérés parce qu'on les a appris. Un croquis intermédiaire doit «être proposé(s) en fonction du sujet ». C'est bien de faire un croquis de Détroit appris dans l'année, mais il ne faut pas le "plaquer" tel quel. Il faut y insérer les minorités et mettre en évidence leurs implications spatiales. « Le croquis doit aider la démonstration, la fluidité et montrer les variations d'échelles " (Duclert, 2014, p. 104). Les deux exemples de schématisation de l'illustration 7 peuvent correspondre à un sujet portant sur les aspects géostratégiques des espaces maritimes, en faisant évoluer si besoin la légende apprise pour la mettre en lien avec la problématique du sujet. Ces deux exemples ne pourraient en revanche correspondre à un sujet portant sur les menaces environnementales qui pèsent sur les milieux marins. L'illustration 9 offre ici un exemple de schématisation simple d'une réserve marine. Ce schéma est facilement mémorisable et reproductible à l'aide d'un compas. Il offre un exemple diversifié en lien avec l'actualité (article paru lors de la COP 21) qui pourrait illustrer une réflexion sur les actions des sociétés locales pour préserver leur ressource halieutique.

Illustration 9 - Un exemple de schématisation simple d'une réserve marine

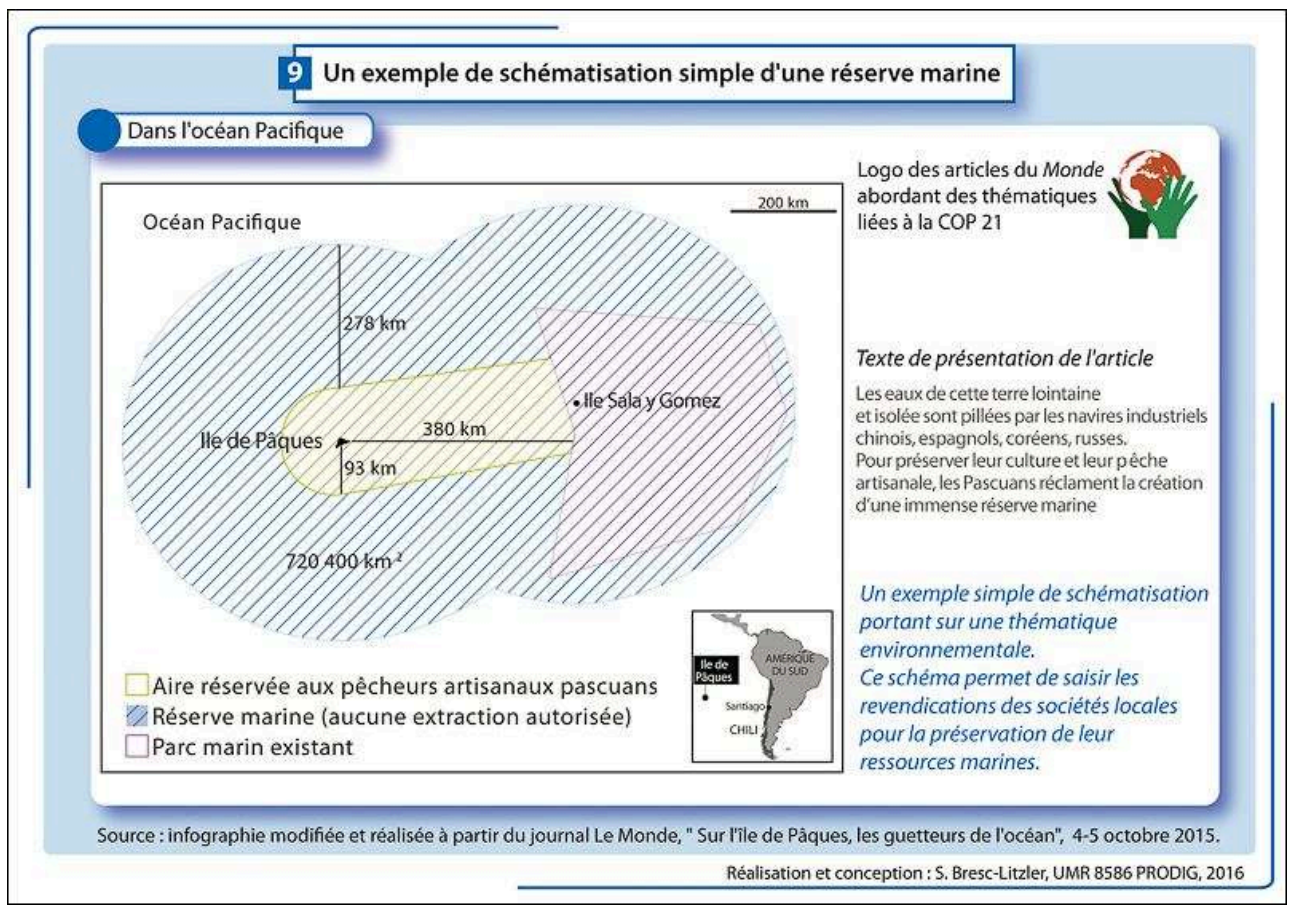

Les croquis intercalaires sont aussi d'autre part des outils pour justifier et consolider une réflexion géographique. Ils servent d'appui pour spatialiser des idées argumentées et fondées à partir de lectures bibliographiques. Ils permettent de faire varier les échelles d'analyse et de nuancer des propos. Les croquis intercalaires insérés dans le cours de la rédaction offre la possibilité au candidat d'aller plus loin dans sa démonstration, de spatialiser sa réflexion et de localiser des phénomènes qui ne seraient pas forcément visibles à petite échelle. Développer un croquis intercalaire et un croquis de synthèse permet de comprendre l'esprit de la géographie et de ne pas se laisser tenter par la multiplication d'exemples cités sans être suffisamment développés 
pour offrir une rédaction problématisée : "Raisonner en géographe ne consiste pas à présenter une série d'exemples plus ou moins en lien avec le sujet. [...] Un exemple en géographie sert, soit à étayer le raisonnement, soit à permettre une généralisation qui donne sens aux idées avancées » (Duclert, 2014, p.117). L'exemple qui « sert à étayer un raisonnement " peut en effet s'appuyer sur un croquis intercalaire avec une légende construite. Ce sont des savoir-faire scientifiques propres à la discipline géographique ; être capable de maîtriser ces techniques, c'est ensuite être capable de les transmettre pédagogiquement aux élèves et de leur montrer que la schématisation et les croquis sont des outils pertinents pour la compréhension du monde ${ }^{28}$.

\section{Conclusion}

Cet article illustre une méthode de travail qui se fonde sur les capacités essentielles des étudiants aux concours. Ces analyses proposées de documents, ces schémas, ces références, ces méthodes et ces conseils sont utiles pour l'acquisition des savoirs dans le cadre de la préparation des grandes questions de géographie au programme pour la session 2017 du CAPES. Nous souhaitons aussi que les infographies réalisées expliquent clairement au candidat les démarches de la réflexion géographique et didactique à suivre dans le cadre des épreuves du concours; les candidats pourront à leur tour exploiter ces méthodes pour d'autres questions et constituer leur propre réserve de schémas et croquis intercalaires. Nous avons pris soin de bien référencer les extraits des rapports du jury et des textes officiels utilisés afin que les candidats puissent s'y référer facilement et y poursuivre leur lecture attentive et rigoureuse des conseils et des descriptions des épreuves donnés par ces rapports des sessions 2014, 2015 et 2016.

Préparer les concours de l'enseignement en histoire-géographie est ainsi un exercice exigeant qui nécessite à la fois une solide culture générale, une maittrise des savoirs disciplinaires, une réflexion sur les pratiques pédagogico-didactiques et une bonne connaissance des programmes du secondaire. Toutes ces compétences sont nécessaires au candidat comme au futur enseignant devant sa classe ou en enseignement interdisciplinaire.

La réforme du collège appelle en effet les professeurs des différentes disciplines à travailler ensemble notamment avec les E.P.I (Enseignements Pratiques Interdisciplinaires). Cette dynamique de l'interdisciplinarité touche particulièrement les enseignants d'Histoire-Géographie et d'EMC. C'est pourquoi nous avons réalisé une dernière infographie (illustration 10) montrant les EPI possibles pour les deux questions de géographie en nous appuyant sur le BOEN spécial n ${ }^{\circ} 1$. 
Illustration 10 - Les EPI la pratique pédagogique de l'interdisciplinarité

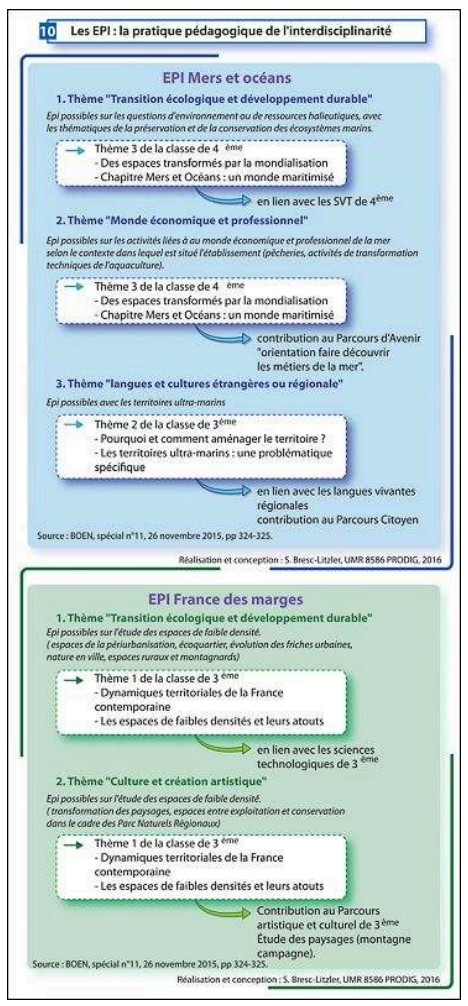

Ce concours s'est donc adapté à l'évolution de l'enseignement. Il l'accompagne et la favorise, tout en gardant la nécessité fondamentale d'être très bien formé scientifiquement et didactiquement pour enseigner dans la société française de demain ${ }^{29}$. Il convient donc aussi de s'adapter à ce nouveau concours pour pouvoir enseigner la géographie en répondant à de nouveaux enjeux didactiques et pédagogiques.

Dans un article de 1982, Paul Pélissier répondait ainsi à la question Pourquoi enseigner la géographie ?: «parce qu'elle apporte un savoir indispensable à l'intelligence du monde contemporain, de ses solidarités, de ses inégalités, de ses conflits (...). Parce qu'elle apprend à hiérarchiser les échelles, à découvrir les valeurs des sociétés différentes, à comparer les situations, à rechercher des explications spécifiques " (Pélissier, 1989). Toutes ces compétences sont toujours importantes aujourd'hui et nécessaires à acquérir pour la mise en œuvre de l'enseignement de la géographie. Continuer à transmettre ces méthodes en classe contribue à faire de la géographie une science « vivante » qui intéresse et fait réfléchir les élèves. Dans un article invitant à penser le renouveau didactique de la géographie Bernadette Mérenne-Schoumaker définit la géographie «vivante » ainsi : " une géographie est vivante quand elle sert à percevoir et à décrire le monde réel en profondeur, quand elle contribue à rechercher des explications sur la façon dont le monde fonctionne et quand elle aide à concevoir des solutions alternatives (...) Elle encourage l'analyse critique de " grandes idées » comme le développement durable, la globalisaton » (Mérenne-Shoumaker, 2016). En enseignant devant les élèves du secondaire, ce sont bien ces principes didactiques qui sont mis en en œuvre au quotidien: enseigner la géographie en s'appuyant sur les outils médiatiques et numériques (par exemple l'utilisation de Google Earth), croiser les sources journalistiques issues des grands médias, aller rechercher sur les sites les 
auteurs de la géographie universitaire (à l'aide de revues disponibles en ligne), montrer aux élèves que ce qu'ils apprennent a été théorisé, conceptualisé par leurs professeurs et des chercheurs, c'est aussi leur donner le goût d'un travail intellectuel rigoureux et sérieux. Ce travail passe par l'emploi de la carte, outil majeur du géographe pour traduire en image sa démonstration scientifique. Leur apprendre à les lire, à les comprendre, à les critiquer et à les construire (dans le cadre des épreuves du croquis de bac) sont des compétences qui s'acquièrent aussi en préparant les concours. Elles seront largement utilisées par la suite dans l'enseignement de la géographie à l'école et montrent les interactions didactiques et pédagogiques entre la géographie universitaire et scolaire. Maîtriser sa discipline en étant capable de penser les transpositions didactiques d'un savoir géographique ouvre aussi la voie à des travaux interdisciplinaires plus poussés. Les enseignements d'exploration SVT-géographie sont de bonnes illustrations des croisements de savoirs entre les disciplines au lycée. Ces enseignements sont l'occasion d'aller parfois plus loin dans les thématiques des programmes. Avec des sujets portant par exemple sur les mises en œuvre scientifiques et biologiques d'une agriculture urbaine; ils offrent la possibilité de montrer concrètement comment nourrir 9 milliards d'hommes. Ces enseignements d'exploration, par la grande liberté qu'ils peuvent laisser aux enseignants, permettent d'intégrer les nouvelles problématiques de la géographie ${ }^{30}$ et faire percevoir aux élèves des nouveaux champs d'intérêt scientifique de la discipline. Ce sont ici quelques pistes de réflexions didactiques qui appellent de plus amples approfondissements en se fondant notamment sur des études plus poussées dans les classes auprès des enseignants en histoire et géographie.

Question « France des marges » : Site de l'ENS de Lyon, Géoconfluences, les grands axes thématiques de la question la France des Marges par L. Carroué et M.-C. Doceul.

\section{BIBLIOGRAPHY}

\section{Références universitaires et didactiques}

Brunet R., 1997. Territoires : art de la découpe. Revue de Géographie de Lyon. Vol. 72-3/97.

Ciattoni A. (dir.), Beucher M., Reghezza M., 2005. La géographie : pourquoi ? Comment ? Objets et démarches de la Géographie d'aujourd'hui. Hatier, collection Initial 288 p.

Granier G., 2013. Les grandes étapes de la géographie scolaire de 1870 à nos jours. Paru en novembre 2013 sur le portail d'Eduscol, 13 p. Disponible en ligne à partir du site de l'E.N.S de géoconfluence : http://geoconfluences.ens-lyon.fr/actualites/veille/didactique-de-lageographie-quelques-ressources-pour-preparer-les-concours-de-lenseignement

Mérenne-Schoumaker B., 2016. Pour un renouveau de la didactique de la géographie. Cybergeo: European Journal of Geography, Les 20 ans de Cybergeo, mis en ligne le 16 août 2016, consulté le 04 novembre 2016. URL : http://cybergeo.revues.org/27746

Mével Y., Tutiaux-Guillon N., 2013. Didactique et enseignement de l'histoire-géographie au collège et au lycée. Edition Publibook Université, Paris, 289 p. 
Pélissier P., 1989. Pourquoi enseigner la géographie? Espace géographique, tome $18, \mathrm{n}^{\circ} 2, \mathrm{La}$ géographie et ses enseignements.

Sierra A., Bonnet-Pineau E., 2016. La réforme territoriale en France : parlementaires et géographes face à « l'art de la découpe » gouvernementale », EchoGéo, 35, 2016. http:// echogeo.revues.org/14481

Tertrais B., Papin D., Laborde X., 2016. Atlas des frontières, murs, conflits, migrations, Les Arènes, $133 \mathrm{p}$.

Themines J.-F., 2016. La géographie du collège à l'épreuve des récits. EchoGéo37 | 2016, mis en ligne le 07 Octobre 2016. http://echogeo.revues.org/14651

Textes officiels disponibles en ligne sur le portail histoire-géographie, eduscol.education.fr. Site du ministère de l'Education Nationale

Rapports de Jury

Dorel G., 2000. Rapport du jury, CAPES externe, session 2000. Historiens et Géographes, $\mathrm{n}^{\circ} 372$, octobre 2000, p. 314.

Duclert V., 2014. Rapport du jury, concours CAPES externe, section Histoire-Géographie, Session 2014 rénovée. Historiens et Géographes, n 428, octobre-novembre 2014.

Duclert V., 2015. Rapport du jury, concours CAPES externe, section Histoire-Géographie, Session 2015. Consulté sur le site Eduscol, paru en août 2015, 65 p.

Duclert V., 2016. Rapport du jury, concours CAPES externe, section Histoire-Géographie, Session 2016. Consulté sur le site Eduscol, paru en septembre 2016, 140 p.

Sujet d'épreuve

Minorités et recompositions territoriales en Amérique du Nord (Canada, Etats-Unis, Mexique) : sujet de l'épreuve 1 à l'écrit du CAPES de la session 2014, Géographie.

Mondialisation, routes maritimes et révolution des transports : sujet de l'épreuve 2 à l'écrit du CAPES de la session 2015, Géographie. Disponible en ligne sur le portail d'histoire-géographie : http:// eduscol.education.fr/histoire-géographie/

Présentation des programmes de la session 2017 du CAPES externe

Programme de la session 2017, Concours externe du CAPES et CAFEP-CAPES, section Histoire et géographie, www. education.gouv.fr, 24 mars 2016.

Bulletin officiel

Bulletin Officiel de l'Éducation Nationale spécial, n 11, 26 novembre 2015, 388 p.

Sitographie

Question « Mers et Océans » : site de l'ENS de Lyon : Géonconfluences, http://geoconfluences.enslyon.fr/informations-scientifiques/dossiers-thematiques/oceans-et-mondialisation/articlesscientifiques

Didactique de la géographie

Site de l'ENS de Lyon, Géoconfluences, Didactique de la géographie : quelques ressources pour préparer les concours de recrutement d'enseignant. http://geoconfluences.ens-lyon.fr/ actualites/veille/didactique-de-la-geographie-quelques-ressources-pour-preparer-les-concoursde-lenseignement

Outils 
Entretien entre l'Amiral Laurent Mérer et Eric Frécon au sujet de l'ouvrage « Moi, Osmane, pirate somalien ». EchoGéo [En ligne], 10 | 2009, mis en ligne le 09 septembre 2009, consulté le 17 juin 2014. http://echogeo.revues.org/11419

Husken D., Bervas A., 2011. Manuel de géographie $1{ }^{\text {ère }}$ L/ES/S. France et Europe dynamiques des territoires dans la mondialisation. Edition Hachette éducation, p. 101, 109, 111.

Janin E., 2014. Manuel de géographie de Seconde. édition Nathan, p. 214-215.

Litzler S., 2012. L'algoculture et ses contraintes géographiques en Chine. EchoGéo [En ligne], 19| 2012, mis en ligne le 10 février 2012, consulté le 17 mars 2016.

UNOSAT, UNITAR, 2014. Global Report on Maritime Piracy a geospatial analysis 1995-2013. 40 p.

\section{NOTES}

1. Duclert V., 2014. Rapport du jury, concours CAPES externe, section Histoire-Géographie, Session 2014 rénovée. Historiens et Géographes $n^{\circ} 428$, octobre-novembre 2014, p. 100. Ce rapport est aussi disponible en ligne à l'adresse suivante : http://eduscol.education.fr/histoiregéographie/.

2. Il existe en effet de nombreuses maisons d'éditions qui demandent à des universitaires, des chercheurs et des enseignants de participer à des ouvrages consacrés aux questions de concours (les auteurs sont souvent des spécialistes de ces questions et des enseignants du secondaire). Par exemple (liste non exhaustive) les éditions CNED/Sedes, Bréal collection amphi histoire et géographie, collection $U$ et cursus horizon d'Armand Colin, les éditions du Temps, Ellipses, Atlande, collection Belin Sup - Capes-Agrégation, Dunod et les PUR et PUF. À ces manuels spécifiques (sommaire construit autour des problématiques et des méthodes du concours, production d'illustrations et de cartes, conseils didactiques d'enseignants) s'ajoutent des revues thématiques utiles issues par exemple de la documentation française avec la « doc photo ».

3. Une épreuve orale qui portait sur l'épistémologie de l'histoire et de la géographie en lien avec les grandes thématiques de l'enseignement dans le secondaire. Lors de cette épreuve, le jury pouvait être amené à poser des questions sur le contenu des programmes du secondaire.

4. Le rapport de la session rénovée 2014 le précise explicitement : «cela nécessite de sa part [le candidat]- outre une solide culture historique et géographique et un intérêt pour ces domaines ", Idem, p 121.

5. Se reporter au passage " la professionnalisation dans les épreuves », dans Duclert, 2015, p. 9.

6. Voir les atlas des éditions Autrement, et les atlas produits par l'émission du Dessous des cartes et plus récemment l'Atlas des frontières par Bruno Tertrais, Delphine Papin et Xemartin Laborde (éditions des Arènes, 2016). ).

7. J.-F. Thémines a ainsi par exemple analysé dans un article récent la notion d'espace dans des livres médiatisés et destinés au grand public à travers son concept de « récits géographiques de pays ». (Thémines, 2016).

8. «Une bonne préparation de l'épreuve d'Analyse de situation professionnelle implique d'étudier les programmes actuels (Histoire, Géographie, Enseignement moral et civique), leur cohérence et leurs objectifs, d'être familiarisé avec les fiches Eduscol et les manuels du secondaire » (Duclert, 2015, p. 8).

9. Duclert., 2015, p. 7. Se reporter également au rapport du jury, session 2016, p. 16.

10. Duclert, 2015, p. 7.

11. Dorel, 2000, p. 314. 
12. Voir aussi : http://geoconfluences.ens-lyon.fr/actualites/veille/didactique-de-la-geographiequelques-ressources-pour-preparer-les-concours-de-lenseignement. Se reporter également à Duclert, 2016, p. 15-18.

13. Pour avoir plus de détails sur la géographie scolaire se reporter à l'article suivant : Granier G., Les grandes étapes de la géographie scolaire de 1870 à nos jours, sur le portail Eduscol via le site de l'ENS Géoconfluences.

14. Site de l'ENS de Lyon Géonconfluences, consultable en ligne: http://geoconfluences.enslyon.fr/informations-scientifiques/dossiers-thematiques/oceans-et-mondialisation/articlesscientifiques. Et pour la question la France des marges : site de l'ENS de Lyon, Géoconfluences, les grands axes thématiques de la question la France des Marges par L. Carroué et M.-C. Doceul. Disponible en ligne Programme de la session 2017, Concours externe du CAPES et CAFEP-CAPES, section Histoire et géographie, www. education.gouv.fr, 24 mars 2016.

15. BOEN, spécial $n^{\circ} 11,26$ novembre 2015, pages consultées; cycle $3: p .175-187$ et cycle $4: p$. 312-330.

16. Le sujet de l'épreuve 2 à l'écrit du CAPES de la session 2015 (géographie : mondialisation, routes maritimes et révolution des transports). Document $3:$ le port de Yansghan, (le plus grand du monde en eaux profondes), baie de Hangzou, Shanghai. Photographie de Y. Artus Bertrand, mai 2012.

17. «Pour parler de la Révolution des Transports, il était possible (et certains candidats l'ont fait) de mettre en valeur cette photo par un croquis de paysage ou un croquis de l'île », p. 39. Et «Un développement sur le port en eaux profondes de Yangshan était attendu par le jury et il était possible ici (dans la partie I. B. Une course au gigantisme des bateaux et des infrastructures) de réaliser un croquis de paysage ou de l'île à partir du document » (Duclert, 2015 p. 41). Le rapport de la session 2016 offre une correction détaillée du sujet de l'épreuve 2 de l'année dernière : « Entre crise et mutations, un système agricole français en recompositions », p. 59 à 84. 18. Dans la figure nous avons mis un nombre restreint d'auteurs dans le volet «Pour aller plus loin » afin de faciliter la lecture du document. Il existe d'autres auteurs mobilisables sur cette entrée. Se reporter à la bibliographie de l'ENS de Lyon.

19. «Une seconde recommandation doit être portée à la connaissance des candidats et des préparateurs. Si la réussite aux épreuves d'admissibilité conditionne l'accès aux épreuves d'admission, il est conseillé de se préparer dès l'écrit aux épreuves d'oral, d'autant mieux que ces dernières relèvent d'une même conception des enjeux de la transmission et de sa mise en œuvre » (Duclert, 2015, p. 10).

20. « Le candidat analyse et explicite les logiques qu'il identifie de la séquence d'enseignement : il présente les axes retenus par l'extrait de programmes et la fiche EDUSCOL, les discute, réfléchit à la logique des programmes les concernant, justifie, éclaire et interroge les choix proposés par le manuel », (Idem, p. 7).

21. «La seconde partie de l'épreuve, dite «Exploitation adaptée à un niveau donné ", est un écrit de synthèse fondé sur « l'analyse critique » précédemment réalisée et visant à la transmission d'un savoir raisonné à une classe ", (Idem, p. 5). Nous invitons également les candidats à consulter la correction détaillée du sujet de l'épreuve 2 de la session 2015 offerte par le rapport du jury, session 2015, Idem, p. 38-44.

22. Idem, p. 10.

23. Par exemple pour la question la France des marges : site de l'ENS de Lyon, Géoconfluences, les grands axes thématiques de la question la France des Marges par L. Carroué et M.-C. Doceul voir l'entrée « des sites d'informations : des ressources à consulter ».

24. UNOSAT, UNITAR, 2014. Global Report on Maritime Piracy a geospatial analysis 1995-2013. 40 p. L'ISEMAR est aussi un institut français qui fournit des données utiles, notamment sur la piraterie. Voir note de Synthèse $\mathrm{N}^{\circ} 128$, Octobre 2010, Piraterie : Perturbation de l'économie maritime? 
25. Entretien entre l'Amiral Laurent Mérer et Eric Frécon au sujet de l'ouvrage « Moi, Osmane, pirate somalien ». Source: «Entretien », EchoGéo [En ligne], 10 | 2009, mis en ligne le 09 septembre 2009, consulté le 17 juin 2014. URL : http://echogeo.revues.org/11419

26. Duclert, 2014, p. 104-105.

27. Les exigences du croquis de synthèse sont présentées dans l'illustration 7. Les règles de réalisation du croquis de synthèse (sémiologie, soin, légende organisée) sont aussi valables pour le croquis intercalaire. Le rapport de la session 2016 offre également des conseils utiles pour comprendre les exigences attendues de la production graphique. Se reporter au rapport p. 80 et suivantes.

28. Les conseils méthodologiques du rapport du jury 2016 confirment cette idée en signalant une «adaptation» de la partie 2 de l'écrit 2 : «Depuis la session 2016, la seconde partie, dite « exploitation adaptée » est centrée sur la production graphique, élément essentiel des compétences attendues en géographie pour un enseignant du secondaire, au collège comme au lycée ». Se reporter aux pages 80 et suivantes dans le rapport du jury 2016.

29. Comme en témoigne cette phrase extrait d'un article du Monde: "ils (les enseignants) doivent, d'abord, être recrutés sur leur capacité à dire et enseigner une histoire intelligente et problématisée. Ils doivent être intellectuellement prêts, car c'est la connaissance scientifique qui leur permet de réfléchir et de faire réfléchir, de mettre en perspective, de comparer faits et évènements, et qui en faits de bons pédagogues ", in Le Monde, 29 septembre 2015, rubrique débats, Retour du roman national à l'école? Article de Iannis Roder, « Enseigner plus d'histoire de France n'est pas du patriotisme éculé ». Nous pourrions ajouter qu'il en va de même pour la formation en géographie.

30. Par exemple les villes du futur peuvent être abordées dans le cadre d'un travail commun avec les professeurs de français en utilisant entre autres des récits littéraires d'anticipation (fictions et récits utopiques). Cette pratique pédagogique ouvre aux élèves d'autres perspectives de réflexion sur la géographie urbaine et ses représentations.

\section{ABSTRACTS}

Cet article vise à établir une synthèse sur les exigences du nouveau concours de l'enseignement (CAPES) d'histoire-géographie ainsi que sur de nouvelles méthodes pour s'y préparer. Les attentes au concours ont évolué avec le temps; il faut donc aussi bien en comprendre les fondamentaux que réfléchir à la méthode, aux connaissances et au suivi de l'actualité afin de construire des schématisations, une culture générale réactualisée et acquérir une bonne connaissance des programmes du secondaire, qui sont des plus-values aux épreuves du concours de l'enseignement. Nous aimerions ainsi proposer des analyses de documents, des schémas, et des débats d'actualité sur les questions de géographie au programme (Mers et océans, la France des marges). Il s'agit de comprendre parallèlement la démarche de travail et les attentes de la réflexion géographique en identifiant les passerelles didactiques entre la géographie scolaire et la géographie scientifique universitaire : cela passe ainsi par la maîtrise des travaux scientifiques des principaux auteurs des questions mais aussi des échelles, des acteurs et des enjeux afin de les transmettre à de jeunes publics. Ces prolégomènes cherchent donc à fournir aux étudiants une méthode de travail efficace pour acquérir certaines compétences essentielles, nécessaires à la préparation des concours et attendues aux épreuves pour enseigner une discipline scientifique. 
INDEX

Mots-clés: Concours de l'enseignement, programme scolaire, didactique, étude de cas, schématisation, géographie scientifique universitaire, géographie scolaire

\section{AUTHOR}

\section{SOPHIE BRESC-LITZLER}

Sophie Bresc-Litzler, litzler.sophie@gmail.com, est agrégée et docteure en géographie. 